\title{
Editorial
}

\section{Medicina Centrada en la Persona}

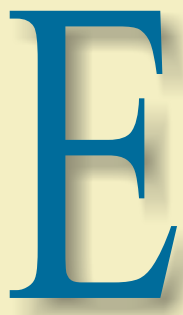

I problema de la medicina

contemporánea.

La medicina, desde sus épocas primigenias, ha sido, es y será una actividad que integra, en diversos porcentajes, arte y ciencia. Por ello, no extraña que en la antigua Grecia fuera categorizada como Thekné, basada en la Doxa (opinión, en este caso experta) y no como ciencia epistémica, con exigencia de exactitud.

La imagen social de la profesión médica era de tal solidez en la antigua Grecia, que la Medicina, junto con el sacerdocio y la política, eran exonerados de responsabilidad ante la sociedad en cuanto a su trabajo. Y por tanto, a diferencia de practicantes de otros oficios, no recibían recompensa pecuniaria por su labor, sino recompensa honorífica, gratitud expresada por el paciente atendido que el médico aceptaba como honorario.

En esta perspectiva, la sociedad siempre apoyó la labor galénica comprendiendo que su quehacer aparte de indispensable- tenía el noble objetivo de ayudar al ser humano, en situación de enfermedad, por vocación de servicio.

Lamentablemente, esta energía de fe generada por su presencia, tan importante para la relación médico -paciente y efectividad terapéutica, fueron debilitándose por conductas inadecuadas de algunos de sus practicantes que tornaron necesarios los códigos éticos de hétero y autocontrol. Hoy apreciamos, con malestar espiritual, que a pesar de los esfuerzos realizados para contener el deterioro de nuestra imagen social, tan vital relación con la sociedad se encuentra en claro conflicto. Se advierten, así, acusaciones de deshumanización al sistema de Atención de Salud, corrupción y mercantilismo. Injerencia de terceras partes vienen alterando la dinámica del acto médico apreciándose una menor mística en muchas instituciones formadoras con visibles intereses de mercado. Y, aunque pudiera parecer exagerado, ya hay voces de alerta frente a riesgos futuros que enfrentará la profesión, pues, con el auxilio del avance tecnológico (inteligencia artificial), algunos creen que con softwares especializados la labor del médico en el proceso diagnóstico y quizá terapéutico, pudiera ser, en el futuro, innecesaria ${ }^{(*)}$.

Aparte de ello, que es ya bastante, la superespecialización de la carrera, con especialistas que solo ven la parte que les corresponde del cuerpo enfermo, cosifican al paciente con virtual desaparición de su condición humana sin conexión con sus circunstancias sociales.

\section{Medicina Centrada en la Persona \\ (MCP) como respuesta.}

Si la verdadera medicina ha integrado siempre ciencia y humanismo en una visión integral de la persona, a punto tal que un distinguido académico sentenciara: "Medicina que no se orienta a la Persona no es medicina" ${ }^{(* *)}$, el problema arriba señalado exige, actualmente, además de una respuesta principista otra más estructurada apoyada en evidencia científica.

Pero la Medicina Centrada en la Persona no nace como tal sino como Programa Institucional de Psiquiatría Centrada en la Persona en la Asamblea General de la Asociación Psiquiátrica Mundial del 2005. Es su desarrollo ulterior que lleva a JE Mezzich a ampliar su límite a toda la medicina.

Explicada en forma muy resumida, la MCP "es una iniciativa mundial que articula la ciencia y el humanismo hacia una medicina de la persona (y su salud total, de la enfermedad a la calidad de vida), para la persona (promoviendo el cumplimiento del proyecto de vida de cada quien), por la persona (cultivando al profesional de la salud como persona, con elevadas aspiraciones éticas y científicas) y con la persona (colaborando respetuosamente con la persona que se presenta en busca de ayuda). Se trata pues de una medicina en la

\footnotetext{
("Comentario realizado por el Dr. Emilio La Rosa (Francia/Perú) en la Conferencia Internacional Sobre Ética y Responsabilidad Social en Salud, Lima
} Noviembre 2018. ${ }^{(*)}$ Comentario realizado por el Académico Alberto Cazorla Talleri. 
cual la ciencia es instrumento esencial y el humanismo su esencia misma"(1).

Sobre tal marco conceptual un grupo de investigadores del International College of Person Centered Medicine, ejecutó un proyecto de investigación para presentar evidencias objetivas del nuevo enfoque. Primeramente revisaron la literatura mundial sobre el tema, general y focalizada en los archivos del International College of Person Centered Medicine. En una Segunda Fase exploraron los datos contenidos en PubMed de la Biblioteca Nacional de Medicina de los Estados Unidos. Generaron, así, un documento que fue enviado a consulta internacional de expertos de los cinco continentes. El resultado permitió al equipo, responsable del proyecto, identificar las áreas claves de la MCP, las cuales, a su vez, permitieron la construcción de un instrumento específico de medición. Las áreas identificadas como esenciales fueron ocho: a) Compromiso ético; b) Marco holístico; c) Sensibilidad y respuesta cultural; d) Foco comunicativo y relacional; e) Individualización del programa de atención clínica; f) Establecimiento de una base común entre clínicos, paciente y familia para el entendimiento y la acción; g) Organización de servicios integrados y centrados en las personas y la comunidad, y h) Educación médica e investigación científica en salud centradas en la persona ${ }^{(2)}$.

\section{Desarrollo de la MCPen el PERÚ y Latinoamérica.}

La MCP, ha tenido presencia oficial en el Perú desde el 2014. Su rápido desarrollo se vincula a la acción de la Red Latinoamericana de MCP apoyada por instituciones claves como la Academia Nacional de Medicina, el Colegio Médico del Perú, el Instituto Nacional de Salud y las universidades Nacional Mayor de San Marcos, Peruana Cayetano Heredia y Católica Santo Toribio de Mogrovejo. Paulatinamente se han agregado diversas Academias Nacionales de Medicina de la Región y, últimamente, la Asociación Latinoamericana de Academias Nacionales de Medicina, España y Portugal (ALANAM) así como la Asociación Peruana de Facultades de Medicina (ASPEFAM) y la recientemente creada Asociación Peruana de Medicina Centrada en la Persona. Toda esta acción se ha traducido en diversas actividades y Jornadas Anuales de trabajo, que siempre han contado con el decidido apoyo y asesoría del International College of Person Centered Medicine,
A propósito, con ocasión de las Terceras Jornadas Latinoamericanas de Medicina Centrada en la Persona, realizadas en Lima, del 16 al 17 de Diciembre de 2017, acompañan este Editorial 6 importantes documentos para fundamentar el presente Simposio:

$\left.1^{\circ}\right)$ El autor del primer artículo: "Educación Médica Centrada en la Persona en el Siglo XXI", Dr. Juan Enrique Mezzich, distinguido psiquiatra peruano radicado en Estados Unidos, líder del movimiento internacional que genera esta publicación y Secretario General del International College of Person Centered Medicine, plantea con sinceridad la realidad actual de la educación médica en los lineamientos de la MCP. La metodología que utiliza para esta contribución nos parece muy pertinente, pues estudia los documentos generados por reuniones de análisis del tema en diferentes escenarios, nacionales e internacionales. De ellas extrae importantes conclusiones, que plantea con mesura y prudencia: A pesar de todos los logros obtenidos, en su concepto, aún falta mucho para poder variar el paradigma biológico-tecnológico centrado en la enfermedad hacia otro más comprehensivo, integral y humanizado.

$2^{\circ}$ ) El segundo artículo: "Formación ética centrada en la perspectiva de la persona: un aporte metodológico", del Académico Titular de la Academia Nacional de Medicina de Uruguay, Dr. Oscar Cluzet, constituye un sesudo análisis y aporte al tema de la Educación Médica. Luego de examinar los antecedentes básicos de la Medicina Centrada en la Persona (MCP) y extrapolando sus principios rectores plantea la necesidad de centralizar la formación ética del profesional de las ciencias de la salud, y particularmente de la medicina, en la dignidad, los derechos humanos y los valores no solo en la persona del paciente sino, también, en la persona del médico. Afirma que sin un encuadre sinceramente humanista y con reconocimiento de los valores y derechos del paciente como persona, pensar en un manejo adecuado de su enfermedad y dolencia resultan inconcebibles. El adecuado proceso del Consentimiento Informado, y respeto por su autonomía, constituyen los pilares de una buena atención clínica centrada en la persona caracterizada por una medicina humanizada sin dejar de apoyarse en las evidencias científicas.

$\left.3^{\circ}\right)$ El tercer artículo: "Educación en epidemiología para una medicina centrada en la persona", presentado por el Dr. Javier E. Saavedra, 
Director Ejecutivo de la Oficina Ejecutiva de Apoyo a la Investigación y Docencia Especializada del Instituto Nacional de Salud Mental "Honorio Delgado - Hideyo Noguchi", es un trabajo original de ideas originales y valiosas sobre el tema. Propone que la enseñanza de la epidemiología integre dos aspectos: la enfermedad y la salud, es decir los factores que inciden en la presencia y desarrollo de la primera y de aquellos salutogénicos que generan bienestar. Del mismo modo debe integrar los períodos pre-patogénico y patogénico así como el período pre-salutogénico y salutogénico, que permitirían entender el cuadro completo del proceso salud -enfermedad en una visión más holística, como si se tratara de un continuo en el cual el ser humano transita de un extremo negativo de enfermedad a uno positivo de bienestar con todas las variantes intermedias imaginables, sometidas a las diversidades contextuales del entorno, tanto a favor de la salud negativa cuanto aquello que genera salud positiva. Un modelo más integrado y comprehensivo de esta naturaleza, dirigido a la totalidad de las personas devendría en una ciencia epidemiológica y en una verdadera epidemiología de la salud.

$\left.4^{\circ}\right)$ El cuarto artículo, "Desarrollo de competencias para la investigación en pre-grado centradas en la persona", es obra del Dr. Fausto Garmendia Lorena, Profesor Extraordinario Experto de la Universidad Nacional Mayor de San Marcos (UNMSM). Su colaboración constituye un logrado aporte que trasunta no solo la vasta experiencia del autor en el área de la investigación científica sino que describe adecuadamente sus dos modalidades: curricular y no curricular. Agrega en su análisis las ventajas formativas que conlleva la utilización del método científico en la adquisición de competencias clínicas y otras deseables en los alumnos de pre-grado, aún en aquellos que no muestran vocación por dedicarse a la investigación como actividad profesional preferente en el futuro.

$5^{\circ}$ ) El quinto artículo, cuyo responsable es autoridad médica nacional en el tema de Desastres y Emergencias, el Dr. Raúl Morales Soto, Presidente de la Academia Nacional de Medicina, constituye un aporte valioso a la comprensión de la centralidad de la persona -no solo en la evaluación de la gente afectada sino también de aquellos que -a pesar de las difíciles circunstancias que implica un desastre natural- deben asistir y dar lo mejor de sí a las víctimas, cuando ellos también son personas que habrán de soportar un alto estrés situacional y de responsabilidad, no infrecuentemente dañinas para su salud. La necesidad de programas de autocuidado y protección del personal de salud especializado en estas circunstancias es de primerísima importancia.

Finalmente, y como corolario necesario para transmitir la riqueza de contenido y experiencia extraídos de las Terceras Jornadas Latinoamericanas de MCP, reunión de las que emanan las contribuciones a este Simposio, anexamos el texto completo de la Declaración de Lima, 2017 allí trabajada, para que el lector tenga el encuadre necesario de la temática tratada.

Termino agradeciendo al Comité Editorial de la prestigiada Revista DIAGNÓSTICO por la oportunidad brindada, así como a todos los profesionales que participaron en el evento citado, deseando que el contenido de este Simposio sirva a los lectores para informarse de un movimiento que reclama la necesidad de volver a integrar el humanismo de la práctica médica con la ciencia pertinente, en un encuadre ético de derechos humanos, tanto para el paciente, para su familia doliente cuanto para el propio personal profesional.

Y señalar que en nuestro criterio, la presencia de la MCP en nuestro país y en Latinoamérica, por su alta carga de pobreza y subdesarrollo, constituye no solo necesidad científica sino, además, responsabilidad ética, para convertir la atención de salud de nuestra región en cuidado responsable de todo ser humano en situación de enfermedad, y ayudarle al logro del máximo bienestar en condición de salud.
Referencias bibliográficas

1.Mezzich JE, Perales A. Atención clínica centrada en la persona: principios y estrategias. Rev Peru Med Exp Salud Pública, 2016, 33 (4):794-800. Doi: 10,17843/rpmesp.2016.334.2567

2.Mezzich JE, Kirisci L, Salloum IM, Adams N, Walleraf J, Trivedi JK et al.
Systematically Conceptualizing Person Centered Medicine and Development and Validation of a Measurement Index. International Journal of Person Centered Medicine. 